\title{
Temporal properties of the lens eyes of the box jellyfish Tripedalia cystophora
}

\author{
Megan O'Connor · Dan-E. Nilsson · Anders Garm
}

Received: 3 August 2009 / Revised: 18 December 2009 / Accepted: 13 January 2010 / Published online: 4 February 2010

(C) The Author(s) 2010. This article is published with open access at Springerlink.com

\begin{abstract}
Box jellyfish (Cubomedusae) are visually orientating animals which posses a total of 24 eyes of 4 morphological types; 2 pigment cup eyes (pit eye and slit eye) and 2 lens eyes [upper lens-eye (ule) and lower lens-eye (lle)]. In this study, we use electroretinograms (ERGs) to explore temporal properties of the two lens eyes. We find that the ERG of both lens eyes are complex and using sinusoidal flicker stimuli we find that both lens eyes have slow temporal resolution. The average flicker fusion frequency (FFF) was found to be approximately $10 \mathrm{~Hz}$ for the ule and $8 \mathrm{~Hz}$ for the lle. Differences in the FFF and response patterns between the two lens eyes suggest that the ule and lle filter information differently in the temporal domain and thus are tuned to perform different visual tasks. The data collected in this study support the idea that the visual system of box jellyfish is a collection of special purpose eyes.
\end{abstract}

Keywords Box jellyfish - Visual systems - Flicker fusion frequency $\cdot \mathrm{ERG} \cdot$ Temporal resolution

\section{Introduction}

Box jellyfish possess an elaborate visual system consisting of 24 eyes of 4 morphological types; 2 pigment pit type

M. O'Connor · D.-E. Nilsson

Department of Cell and Organism Biology,

Lund University, Lund, Sweden

\section{A. Garm ( $₫)$}

Department of Comparative Zoology,

University of Copenhagen,

Universitetsparken 15, 2100 Copenhagen, Denmark

e-mail: Algarm@bio.ku.dk eyes (pit eye and slit eye) and 2 lens eyes [upper lens-eye (ule) and lower lens-eye (lle)], which are structurally reminiscent of vertebrate and cephalopod eyes (Fig. 1b). When considering the relatively sparse nervous system of box jellyfish (Parkefelt et al. 2005; Garm et al. 2006; Skogh et al. 2006), combined with the complexity of their visual system, the question arises: how do the eyes of box jellyfish filter and process visual information?

The temporal filtering properties of a visual system greatly influence visual acuity and the quality of an image in a moving world (Srinivasan and Bernard 1975). Consequently, fast moving animals tend to have faster temporal resolution than slower ones (Howard et al. 1984; Laughlin and Weckström 1993). Additionally, temporal resolution influences the quality of an image as it impacts on a system's photon capture and therefore influences the system's signal-to-noise ratio. For this reason, slower temporal resolution is an adaptation often seen in animals living under dim light conditions (Warrant 2004).

Garm et al. (2007a) made preliminary observations of temporal filtering in the lens eyes of the Caribbean cubomedusa Tripedalia cystophora (Fig. 1a). In this initial study, Garm et al. (2007a) found that in response to a flash of white light $\left(3.2 \times 10^{5} \mathrm{~W} / \mathrm{sr} / \mathrm{m}^{2}\right)$, the minimum average half-width of the impulse response was $30 \pm 1.4 \mathrm{~ms}$ (mean \pm SEM) and $27 \pm 0.7 \mathrm{~ms}$ (mean \pm SEM) for the ule and lle respectively. Surprisingly, flash duration (5-100 ms were tested) had little or no effect on the half-width. These half-widths are comparable to those of the nocturnal flying bee Megalopta genalis which had an average half-width of $32 \mathrm{~ms}$ (Warrant et al. 2004). Medusae of T. cystophora are found reliably during the day under the mangrove canopy, amongst the prop roots (Stewart 1996; Buskey 2003) and is an active and agile swimmer. Visually orientated behaviours observed for $T$. cystophora include obstacle 

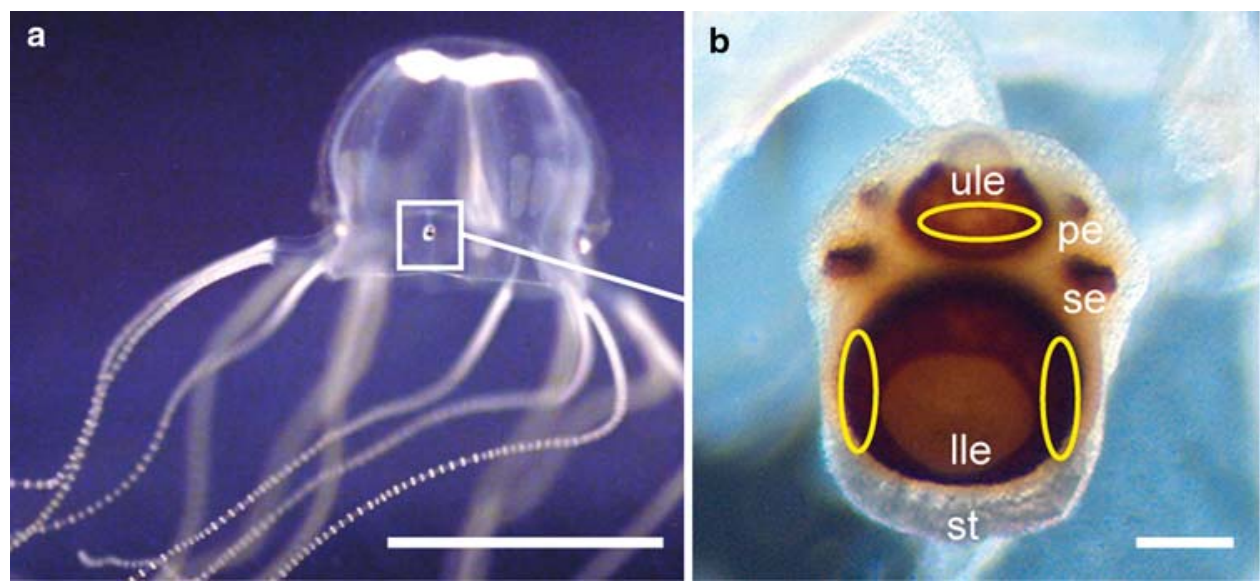

Fig. 1 The visual system of box jellyfish. a A mature Tripedalia cystophora medusa with a rhopalium highlighted (white box). Each medusa possesses four rhopalia, each located midway between two pedalia. Scale bar $1 \mathrm{~cm}$. b Each rhopalium carries four morphologically distinct eye types; two pigment cup type eyes (pit-eye, $p e$, and slit

avoidance (Garm et al. 2007b), orientation to light shafts (Buskey 2003) and optimizing time within light shafts (Buskey 2003; Garm and Bielecki 2008). The half-widths measured for T. cystophora were strongly dependent on the intensity of the light flash: a decrease in flash intensity increased the half-width (Garm et al. 2007a), however, these response dynamics did not change with adaptation state, in contrast to the responses of most other photoreceptor cells (Howard et al. 1984).

In the present study, we seek to gain a better understanding of the response properties and temporal resolution of the lens eyes in T. cystophora. We present sinusoidal light stimuli of varying frequencies to the lens eyes and determine the flicker fusion frequency (FFF), e.g. the maximum frequency, which the eye can resolve from ERG recordings. We find that the temporal resolution of both the ule and lle is relatively slow but that the response properties differ between the lens eyes, supporting our hypothesis that the visually guided behaviours of box jellyfish are driven by information from a collection of special purpose eyes.

\section{Materials and methods}

Animals and electrophysiological preparation

Medusae used in this investigation were cultured at the University of Copenhagen. Mature medusae with bell heights of 6-10 $\mathrm{mm}$ were used. Rhopalia were cut off at the stalk from the jellyfish bell, and then transferred to a small Petri dish containing sea water. The sea water was kept at a temperature of $28^{\circ} \pm 0.5^{\circ}$ using a Peltier element. A glass suction electrode was attached to areas of the ule or lle eye, se) and two lens eyes (upper lens-eye, ule and lower lens-eye, lle). A crystalline statocyst (st) lies at the base of the rhopalium. Yellow rings indicate the areas where electrodes were attached for electrophysiological recordings. Scale bar $100 \mu \mathrm{m}$

(Fig. 1b) and suction was applied until a small amount of screening pigment was observed entering the electrode (in box jellyfish the photoreceptors contain the screening pigment). The reference electrode was placed in the surrounding seawater. A flash of light was given to test the quality of the attachment.

The recorded signal was filtered by a $50 \mathrm{~Hz}$ notch filter and a high and low pass filters of 0.1 and $1,000 \mathrm{~Hz}$, respectively, and amplified 1,000 times (model 1700 differential AC Amplifier, A-M Systems Inc., Sequin, WA, USA). The signals were recorded with National Instruments data acquisition card DAQCard-NI6229 and LabVIEW 8.5 software (National Instruments, Austin, TX, USA). Light from a cyan Luxeon III star LED (LXHL-LE3C, dominant wavelength $505 \mathrm{~nm}$, Lumileds Lighting, LLC, San Jose, CA, USA) was presented to the eyes via a $500 \mu \mathrm{m}$ light guide. The relatively large diameter of the light guide was used to ensure a maximally even illumination of the retina. Peak transmission of the LED corresponded closely to the maximum spectral sensitivity of the lens eyes (Coates et al. 2006; Garm et al. 2007a). The LED was powered by a custom made amplifier, and controlled by the data acquisition card mentioned above.

\section{Electrophysiological protocol}

Flicker ERG recordings were made from ten ule and ten lle. Only one rhopalium per animal and one eye per rhopalium was used to optimize the quality of the preparation. The sinusoidal stimulus was presented to the eyes in frequency span of $0.5-11 \mathrm{~Hz}$ in $0.5 \mathrm{~Hz}$ steps and for a period of $10 \mathrm{~s}$. Since rectangular pulses consist of many frequencies and sinusoidal stimuli consist of one frequency only, sinusoidal 
stimuli were used in this study to obtain a more accurate frequency analysis. The intensity span of the sinusoidal stimulus was $1.2 \times 10^{1}$ to $4.6 \times 10^{4} \mathrm{~W} / \mathrm{sr} / \mathrm{m}^{2}$, which gave a peak-to-peak contrast of $\sim 1$ (Michelson contrast $\left(L_{\max }-\right.$ $\left.L_{\min }\right) /\left(L_{\max }+L_{\min }\right) ; L=$ luminance $)$. The eyes were adapted to the mean intensity $\left(2.3 \times 10^{4} \mathrm{~W} / \mathrm{sr} / \mathrm{m}^{2}\right)$ for $2 \mathrm{~min}$ before the beginning of the protocol and there was a $30 \mathrm{~s}$ interval between each individual recording also at mean light intensity. The sinusoidal stimuli began at a phase of $270^{\circ}$. Additionally, before and after the series of flicker stimuli was presented, rectangular pulses of $4.6 \times 10^{4} \mathrm{~W} / \mathrm{sr} / \mathrm{m}^{2}$ and varying durations $(10 \mathrm{~ms}-3 \mathrm{~s})$ were presented to the eyes to test and monitor the quality of the preparation. If large differences in the responses to rectangular pulses at the beginning and end of a series were seen, the series was omitted from analysis. The duration of an entire protocol was approximately $30 \mathrm{~min}$. Intracellular electrophysiological recordings were fastidiously attempted, however, were not successful.

\section{Analysis}

Before analysis all recordings were smoothed with a running mean of 11 points (sample rate was $1,000 \mathrm{~Hz}$ for the ule and $10,000 \mathrm{~Hz}$ for the lle). For frequency analysis, a Fast Fourier Transform was carried out for each recording. Tuning curves were then constructed using the power of the fundamental frequency. Average tuning curves were obtained from normalized individual tuning curves.

\section{Results}

General observations

Representative flicker-ERGs from the ule and lle are shown in Figs. 2 and 3 respectively. As illustrated, the responses of both eye types show high levels of non-linearity and differ significantly over the range of frequencies tested. For the low frequencies $(0.5$ and $1 \mathrm{~Hz})$ both eye types responded transiently and returned to the baseline potential for a large portion of the stimuli's cycle (Figs. 2, 3). The initiation of the transient response for the flicker-ERGs was before the stimulus minimum in the ule and usually at the stimulus minimum in the lle. The timing indicates that at least part of the response is initiated by a decrease in intensity. However, as the responses are generally multi-phasic and are still occurring at the rise of the stimulus, they are also likely to contain a response to an increase in intensity. In the remainder of the paper we will refer to a response correlated to an increase in stimulus intensity as $\mathrm{ON}$ response and to a response correlated to a decrease in stimulus intensity as an OFF response.

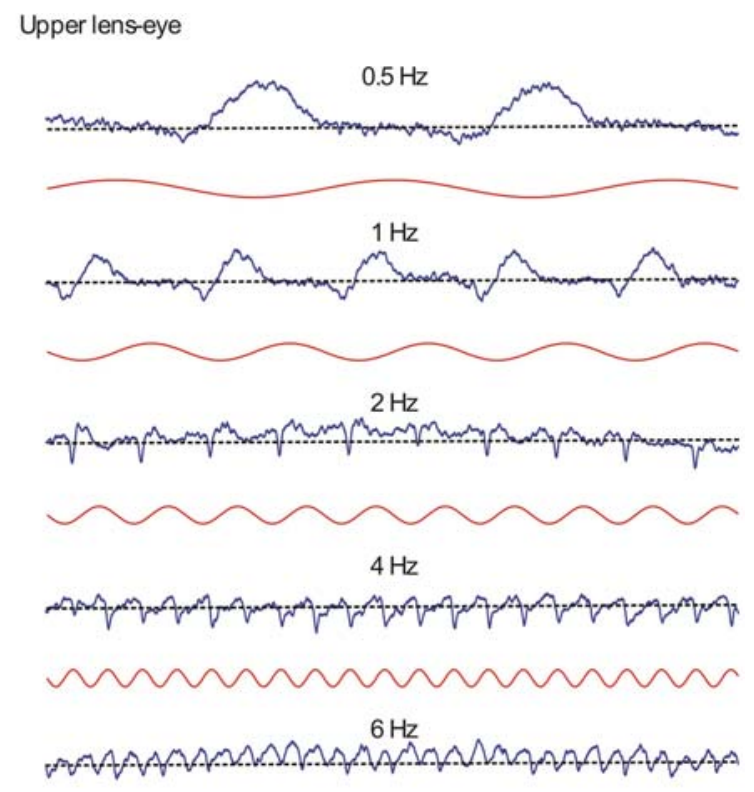

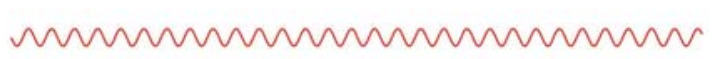

$10 \mathrm{~Hz}$
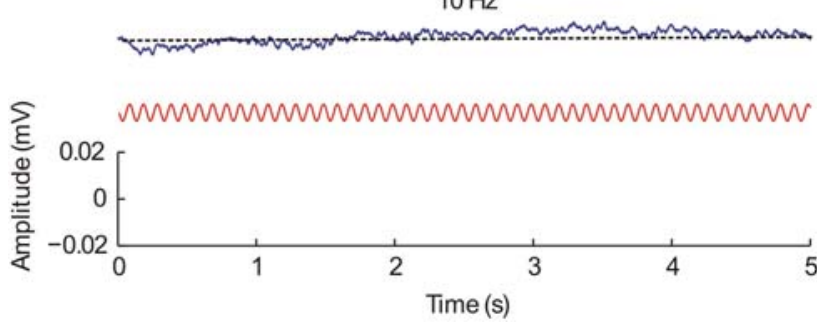

Fig. 2 Representative flicker-ERGs (blue traces) of the upper lenseye to sinusoidal light stimuli (red traces) of selected frequencies. As illustrated, the response signal is complex and differs for different frequencies. At the lowest frequencies tested, the response has a transient pattern. The average FFF for the upper lens-eye was between 9.5 and $10 \mathrm{~Hz}$. The last $5 \mathrm{~s}$ is shown in all recordings. The broken black line indicates the baseline in the recordings. Note that in the 2 and $6 \mathrm{~Hz}$ recordings low frequency noise shift the entire recording away from the baseline

The responses to the rectangular stimuli were similar to earlier results (Garm et al. 2007a). All responses consist of a fast initial peak, considered the receptor response, and often but not always a slower response (Fig. 4). When present the shape of the slower component of the response remained constant within a preparation (Fig. 4a) but varied between preparations (Fig. 4b) and we believe it originates from higher order neurons. Interestingly, within the same preparation, the ON response to rectangular stimuli of longer duration (1 or $3 \mathrm{~s}$ ) was transient and practically identical to the $\mathrm{ON}$ response to short flashes of light (25 or $100 \mathrm{~ms}$ ) (Fig. 4a, inset).

Upper lens-eye: specific response characteristics

The ule generally responded with a negative polarity when presented with flashes of light. Here it should be noted that 


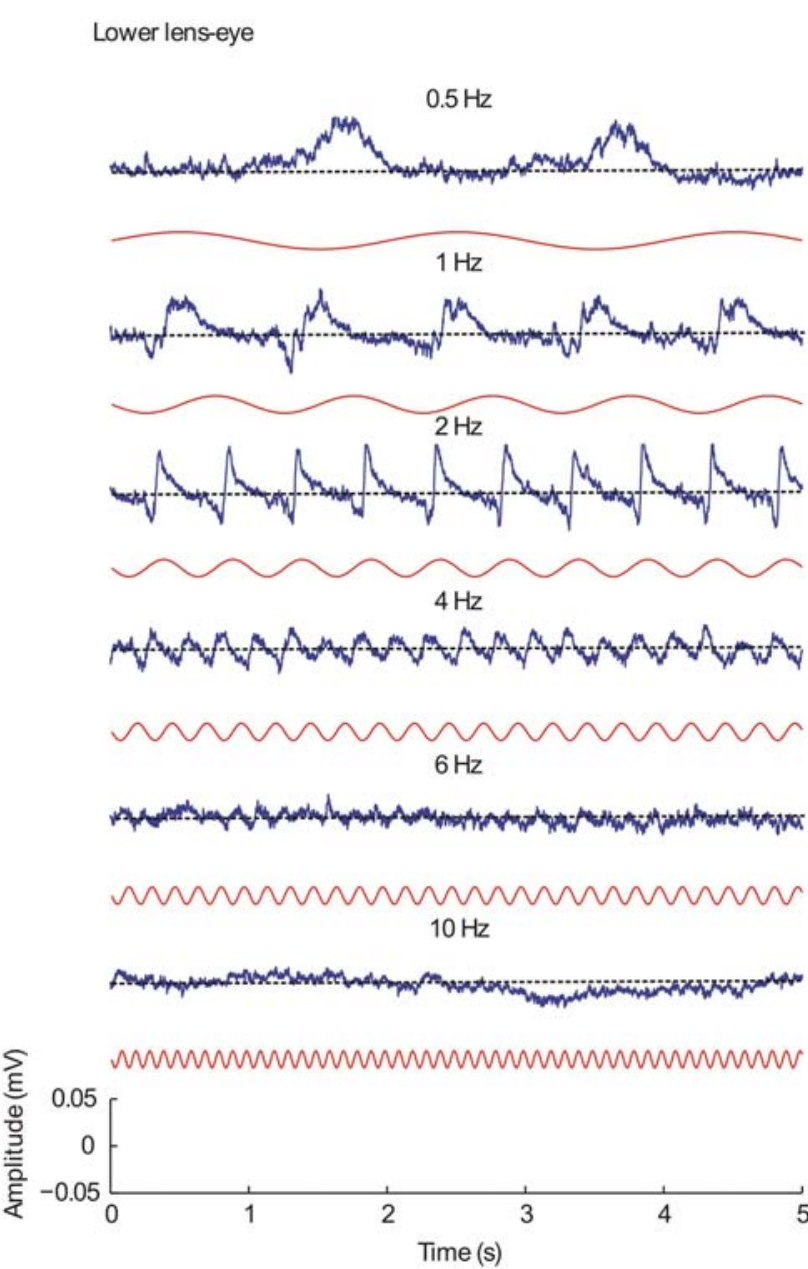

Fig. 3 Representative flicker-ERGs (blue traces) of the lower lenseye to sinusoidal light stimuli (red traces) of selected frequencies. Like the upper lens-eye, the flicker-ERGs of the lower lens-eye are complex. Additionally, at the lowest frequencies, the responses are transient and then as the frequency increases, the responses become more sinusoidal. The average FFF for the lower lens-eye was between 7.5 and $8 \mathrm{~Hz}$, lower than for the upper lens-eyes. Note that the last $5 \mathrm{~s}$ of the response is shown, thus no facilitation is illustrated. The broken black line indicates the baseline in the recordings

the polarity of an ERG says little about polarity of the receptor response and in other cnidarian eyes the ERG polarity has been shown to change with the exact area of attachment (Weber 1982a, b). When presented with stimuli of longer duration, an OFF response, usually opposite in polarity to the $\mathrm{ON}$ response, was often seen with a latency of approximately $0.1-0.5 \mathrm{~s}$. Interestingly, at different frequencies, different types of responses seemed to dominate. For frequencies of 0.5 and $1 \mathrm{~Hz}$, the OFF response appears to dominate the flicker-ERG (Fig. 5a) while, as frequencies increase, up to $4 \mathrm{~Hz}$, the $\mathrm{ON}$ response becomes the dominant part of the response (Fig. 5b). Above approximately $4 \mathrm{~Hz}, \mathrm{ON}$ and OFF responses can no longer be separated (Fig. 2).
From the normalized power spectra, a temporal tuning curve was constructed (Fig. 6a). The tuning curve for the ule has its maximum at $0.5 \mathrm{~Hz}$, a dip at $2 \mathrm{~Hz}$ and then peaks again at approximately $4.5-5 \mathrm{~Hz}$. The FFF (the frequency when no significant peak at the fundamental frequency was detected in the power spectrum) was between 9.5 and $10 \mathrm{~Hz}$.

Lower lens-eye specific characteristics

In contrast to the ule, the initial ON response for the lle was usually positive in polarity when presented with rectangular stimuli (Fig. 4). OFF responses, opposite in polarity to the ON response, and with latencies similar to that of the ule were also often seen for the lle when presented with rectangular stimuli of longer duration. The lle tuning curve was bell-shaped, unlike that of the ule. It had a peak frequency at approximately $2.5 \mathrm{~Hz}$ and a FFF of $7.5-8 \mathrm{~Hz}$ (Fig. 6b). As indicated by the standard error of mean, there was less variability between tuning curves for the lle than the ule.

In contrast to the ule facilitation was regularly observed in the lle for frequencies of approximately $0.5-3 \mathrm{~Hz}$ with the strongest facilitation occurring around approximately $2 \mathrm{~Hz}$ (Fig. 7). To quantify facilitation, peak-to-peak measurements were taken from the first stimulus cycle onwards. At $2 \mathrm{~Hz}$, the response amplitude doubled over the first 4 cycles $(n=10)$ (Fig. 7). At $3 \mathrm{~Hz}$, the amplitude of the response to the second stimulus was smaller than to all other responses in the series. At $4 \mathrm{~Hz}$, the response to both the second and third stimuli was smaller than the responses to the first and subsequent stimuli. Hence, suppression of early responses appears to occur at frequencies of 3 and $4 \mathrm{~Hz}$. At frequencies of $5 \mathrm{~Hz}$ and higher, strong adaptation was seen.

\section{Discussion}

This study shows that the flicker-ERGs of both the ule and lle in T. cystophora are complex and possess non-linearities. Complex responses are often seen in flicker-ERGs and, in some systems, component analysis has been performed using pharmacology and genetics and by comparing the ERG response at different locations (Ruck 1961a; Bush and Sieving 1996; Kondo and Sieving 2001; Krishna et al. 2002; Viswanathan et al. 2002). Such analyses have found that the photoreceptors are the major contributors to the fundamental component of the flicker-ERG's power spectrum determined with FFT while non-photoreceptor responses are the main contributors to the second and higher harmonic components (Baker et al. 1988; Burns et al. 1992; Viswanathan et al. 2002). Additionally, in other 


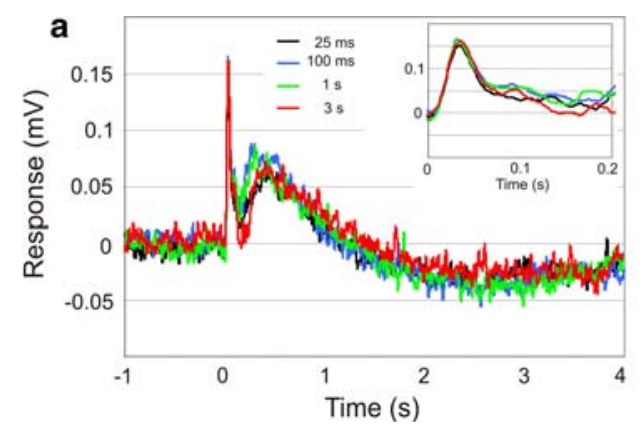

Fig. 4 Responses to rectangular stimuli. Both short (25 and $100 \mathrm{~ms}$ ) and longer ( 1 and $3 \mathrm{~s}$ ) rectangular pulses were presented to the eyes. Responses are here shown for the lower lens-eye only, but transient responses were also obtained from the upper lens-eye although normally opposite in polarity. a Within a preparation all stimuli produced similar transient responses independent of stimulus duration. Inset indicates a close up of the first $0.2 \mathrm{~s}$ of the ON response, demonstrating the similarity in the photoreceptor response. b Between different preparation

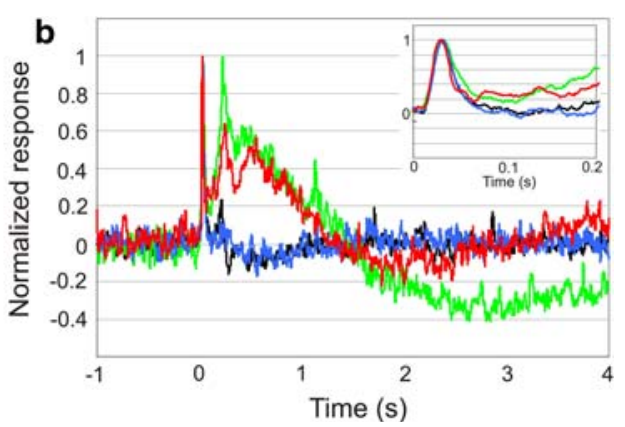

using the same stimulus duration (here $1 \mathrm{~s}$ ) the initial $\mathrm{ON}$ response are also very similar but the slower part varies greatly. Inset indicates a close up of the first $0.2 \mathrm{~s}$ of the ON response, demonstrating the similarity in the photoreceptor response. Note that in some of the preparations (blue and black trace) the slow component is missing and the response returns to the baseline shortly after the ON response. Stimulus on-set is at $T=0$
Fig. 5 Characteristics of the flicker-ERG for different frequencies. a An example of a response of the upper lens-eye to a $0.5 \mathrm{~Hz}$ sinusoidal stimulus. Notice that the signal is dominated by an OFF response. b At approximately $2 \mathrm{~Hz}$, the response pattern of the upper lens-eye ERG changes dramatically and is now dominated by an $\mathrm{ON}$ response
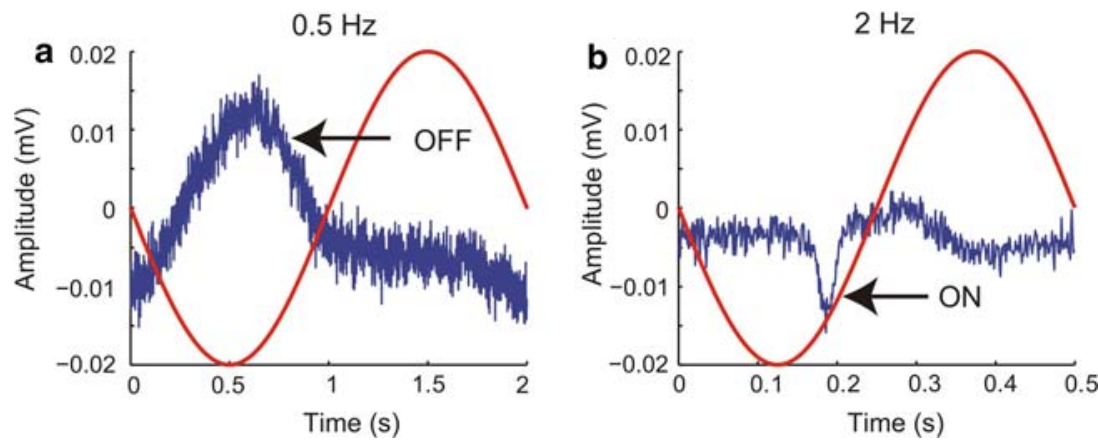

systems, changes in the flicker-ERG across a frequency span have been due to varying contribution of and interaction between second order visual neurons (Ruck 1961b; Kondo and Sieving 2001). From morphological studies it is known that the photoreceptors of $T$. cystophora are in synaptic contact with each other (Gray et al. 2009) while they are also in contact with the rhopalial neuropil via basal neurites (Ekström et al. 2008). Unfortunately, nothing is known about the nature of these connections and it is not possible to perform component analysis on the ERG of box jellyfish. However, the studies mentioned above show that the photoreceptors are the most probable source of the fundamental component of the flicker-ERG and therefore the power of the fundamental frequency was used to construct the tuning curves.

At the lowest frequencies tested, the flicker-ERGs had a transient response pattern. This transient pattern was also clearly seen when the eyes were presented with prolonged, rectangular, light stimuli. Since the $0.1 \mathrm{~Hz}$ of the amplifier's high-pass filter is far below the duration of both the rectangular flashes and the longest sine waves, the transient responses cannot be caused by the amplifier's filtering. Local capacitances can make extracellular recordings differ significantly from the original signals. Such capacitances will differ greatly with each different attachment, and therefore unlikely to generate the similar shaped ON responses reported here (Fig. 4b). Finally, the transient responses could be caused by the stimuli saturating the receptors, but previously experiments on the same eyes used intensities close to a log unit higher with no sign of saturation (Garm et al. 2007a). We therefore suggest that the transient response pattern reflects the activity of the photoreceptors and thus indicates the presence of ON and OFF pathways. If so, it suggests that the visual system of box jellyfish adapts very quickly and is tuned to changes in intensities only and fails to code for steady state light intensities. Still, to make any final conclusions the transient responses have to be verified by intracellular or patch clamp recordings.

Temporal resolution varies greatly throughout the animal kingdom due to differences in lifestyle, habitats and evolutionary origin. At the fast end of the spectrum are fast moving, diurnal insects which can have a FFF of 200 $300 \mathrm{~Hz}$ (Autrum 1958). Such high temporal resolution allows high visual acuity while moving quickly as well as detecting fast moving objects. Low temporal resolution is often seen in slow moving animals living in dim-light 

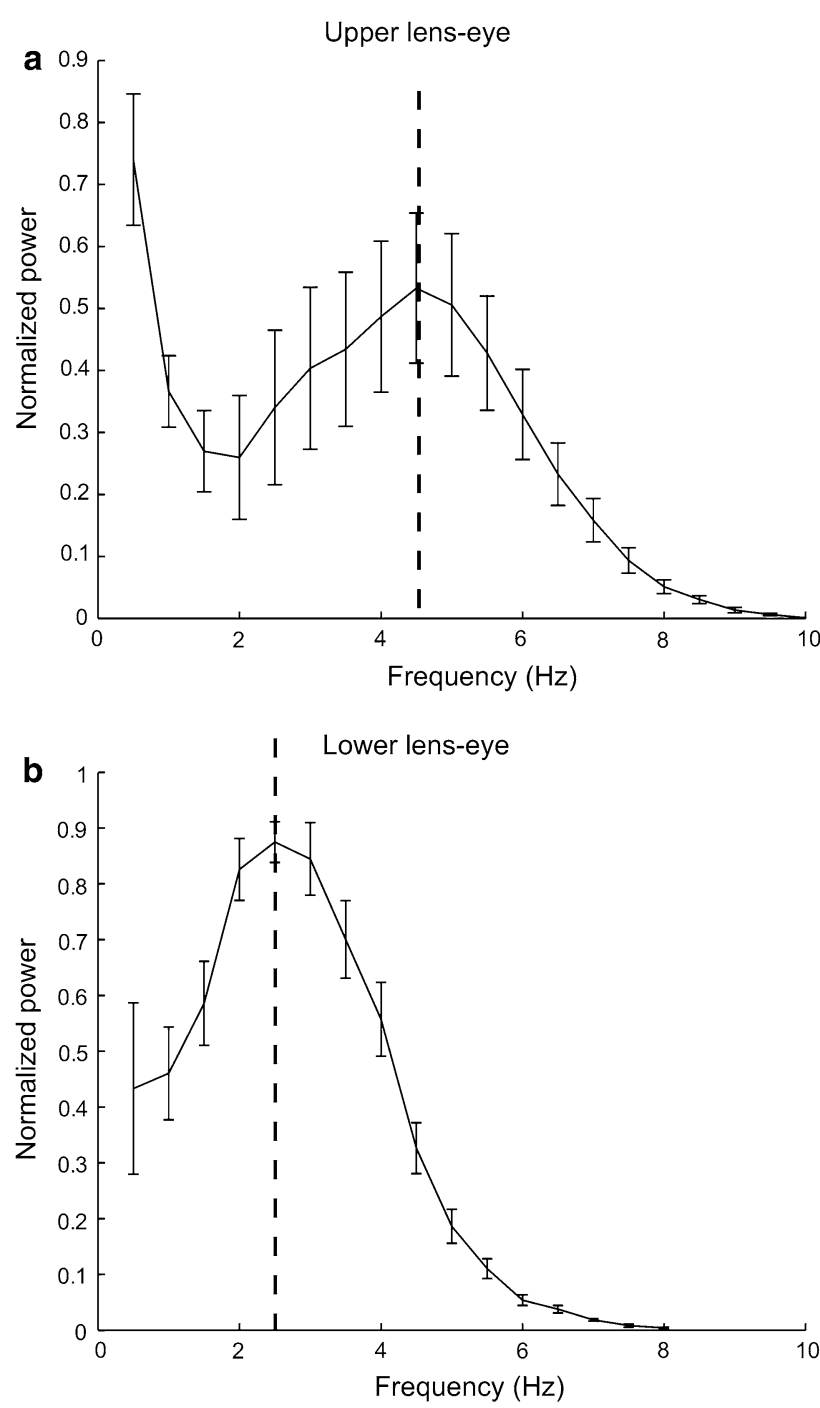

Fig. 6 Temporal tuning curves of the upper and lower lens-eye are calculated from the power of the fundamental frequencies determined by Fourier transformation. Each point is the average of ten eyes where the values have been normalized within each eye. Error bars indicate the standard error of mean. a The tuning curve of the upper lens-eye reveals that there is a dip at approximately $2 \mathrm{~Hz}$ and a peak around 4.5$5 \mathrm{~Hz}$. $\mathbf{b}$ The tuning cure of the lower lens-eye is bell-shaped and peaks at approximately $2.5 \mathrm{~Hz}$

conditions as it improves the signal-to-noise ratio and thus produces a more reliable image. The extreme case is the toad Bufo bufo with integration times up to $1.5 \mathrm{~s}$ (Aho et al. 1993). As mentioned earlier, T. cystophora is diurnal and an active and agile swimmer, able to make quick and effective turns of $120-180^{\circ}$ in less than $0.5 \mathrm{~s}$ (Garm et al. 2007b). Despite this, we find that box jellyfish have relatively low temporal resolution (FFF of 10 and $8 \mathrm{~Hz}$ for the ule and lle respectively). Such low temporal resolution would normally result in a severe reduction in visual acuity. However, in T. cystophora where the half widths of the receptive fields are $10-20^{\circ}$ (Nilsson et al. 2005) the low
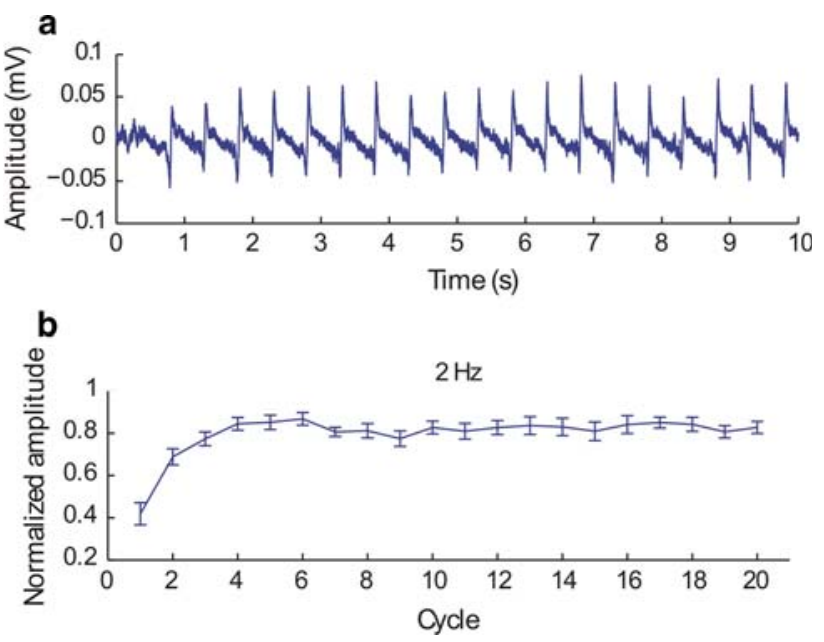

Fig. 7 Facilitation in the flicker-ERG of the lower lens-eye for frequencies between 0.5 and $3 \mathrm{~Hz}$. Facilitation was strongest at $2 \mathrm{~Hz}$ where the response amplitude doubled over the first four cycles $(n=10)$. a An example of a flicker-ERG for $2 \mathrm{~Hz}$ showing facilitation of the response. $\mathbf{b}$ The normalized response amplitude of ten flickerERGs at $2 \mathrm{~Hz}$ showing the steep increase in the amplitude over the first four stimuli cycles. Error bars represent the SEM

temporal resolution will have little effect on visual acuity in most situations. Rather, we believe that the low temporal resolution functions as an important low pass filter.

Low-pass filtering, both in the temporal and spatial domain, agrees with the known visually guided behaviours of box jellyfish. As mentioned previously, visually guided behaviours observed in $T$. cystophora include obstacle avoidance (Garm et al. 2007b), orientation to light shaft (Buskey 2003) and optimizing time within light shafts (Buskey 2003; Garm and Bielecki 2008). All of these behaviours use stationary and relatively large visual cues and hence, would not require high temporal resolution when the jellyfish is not quickly turning. Visual behaviours requiring fast temporal resolution such as predator detection and avoidance and prey detection and capture are not known from box jellyfish. The spatio-temporal low-pass filters therefore removes unwanted small and fast object only, which probably result in an essential optimization of the visual processing.

The low temporal resolution can also be used to remove luminance noise. The medusae of $T$. cystophora are found in the shallow water areas (Stewart 1996) where luminance noise (so-called flicker) is caused by surface-ripples making object detection harder (Maximov 2000). Colour vision can eliminate luminance noise because a colour signal is a comparative response and therefore, to a large extent, insensitive to intensity fluctuations. Consequently, and as proposed by Maximov (2000), flicker may have been a major evolutionary driving force for colour vision in shallow water living animals. The lens eyes of box jellyfish contain only one visual pigment, though, (Coates et al. 
2006; Garm et al. 2007a; Ekström et al. 2008; Koyanagi et al. 2008; Kozmic et al. 2008) making it unlikely that box jellyfish eliminate luminance noise through colour vision. Instead, the low temporal resolution combined with their large receptive fields of the lens-eye receptors (Nilsson et al. 2005) may create a low-pass spatio-temporal filter sufficient to remove luminance noise. Examination of the temporal frequencies experienced during normal behaviour in the natural habitat by $T$. cystophora is needed to resolve the issues of temporal filtering in the visual system of this species.

Interestingly, temporal filtering differs between the ule and lle as demonstrated by the differences in both FFF values and peaks of the tuning curve. Additionally, the orientation of ule and lle on the rhopalia ensures that they are directed to different regions of the visual environment. This strongly indicates that the ule and lle are adapted to different elements of the visual environment and thus dedicated to different visual tasks. To date, no visual task has been identified for the ule, making it difficult to interpret the functional significance of the slightly higher temporal resolution found here. The differences between the ule and lle nonetheless support our hypothesis that the visual system of box jellyfish is a collection of special purpose eyes (Land and Nilsson 2006). The limited number of tasks for such eyes allow for heavy filtering already in the periphery of the visual system. The more filtering performed at the periphery, the less subsequent neural computation is required. In box jellyfish we find heavy filtering of visual information in both the spatial (Nilsson et al. 2005; Garm et al. 2008) and in the temporal domain, as shown here. Hence, the presence of special purpose eyes likely explains how box jellyfish are able to uphold a relatively sophisticated visual system with their comparatively sparse nervous system.

Acknowledgments We appreciate the constructive comments on the MS offered by members of the Vision Group, Lund University. A.G. acknowledges grant \# 272-07-0163 from the Danish Research Council (FNU) and D.-E.N. likewise acknowledges grant \# 2008-3503 from The Swedish Research Council.

Open Access This article is distributed under the terms of the Creative Commons Attribution Noncommercial License which permits any noncommercial use, distribution, and reproduction in any medium, provided the original author(s) and source are credited.

\section{References}

Aho AC, Donner K, Hydén C, Larsen LO, Reuter T (1993) Visual performance of the toad (Bufo bufo) at low light levels: retinal ganglion cell responses and prey-catching accuracy. J Comp Physiol A 172:671-682

Autrum H (1958) Electrophysiological analysis of the visual system in insects. Exp Cell Res 5:426-439
Baker CLJ, Hess RR, Olsen BT, Zrenner E (1988) Current source density analysis of linear and non-linear components of the primate electroretinogram. J Physiol 407:155-176

Burns SA, Elsner AE, Kreitz MR (1992) Analysis of nonlinearities in the flicker erg. Optom Vis Sci 69:95-105

Bush RA, Sieving PA (1996) Inner retinal contributions to the primate photopic fast flicker electroretinogram. J Opt Soc Am A 13:557565

Buskey EJ (2003) Behavioral adaptations of the cubozoan medusa Tripedalia cystophora for feeding on copepod (Dioithona oculata) swarms. Mar Biol 142:225-232

Coates MM, Garm A, Theobald JC, Thompson SH, Nilsson DE (2006) The spectral sensitivity in the lens eyes of a box jellyfish, Tripedalia cystophora. J Exp Biol 209:3758-3765

Ekström P, Garm A, Pålsson J, Vihtlec T, Nilsson DE (2008) Immunohistochemical evidence for several photosystems in box jellyfish using opsin-antibodies. Cell Tissue Res 333:115-124

Garm A, Bielecki J (2008) The swim pacemakers of box jellyfish is modified by the visual input. J Comp Physiol A 194:641-651

Garm A, Ekström P, Boudes M, Nilsson DE (2006) Rhopalia are integrated parts of the central nervous system in box jellyfish. Cell Tissue Res 325:333-343

Garm A, Coates MM, Seymour J, Gad R, Nilsson DE (2007a) The lens eyes of the box jellyfish Tripedalia cystophora and Chiropsalmus sp. are slow and color-blind. J Comp Physiol A 193:547-557

Garm A, O'Connor M, Parkefelt L, Nilsson DE (2007b) Visually guided obstacle avoidance in the box jellyfish Tripedalia cystophora and Chiropsella bronzie. J Exp Biol 210:3616-3623

Garm A, Anderson F, Nilsson DE (2008) Unique structure and optics of the lesser eyes of the box jellyfish Tripedalia cystophora. Vision Res 48:1061-1073

Gray GC, Martin VJ, Satterlie RA (2009) Ultrastructure of the retinal synapses in Cubozoans. Biol Bull 217:35-49

Howard J, Dubs A, Payne R (1984) The dynamics of phototransduction in insects. J Comp Physiol A 154:707-718

Kondo M, Sieving PA (2001) Primate photopic sine-wave flicker erg: vector modeling analysis of component origins using glutamate analogs. Invest Ophthalmol Vis Sci 42:305-312

Koyanagi M, Takano K, Tsukamoto H, Ohtsu K, Tokunaga F, Terakita A (2008) Jellyfish vision starts with cAMP signaling mediated by opsin-Gs cascade. Proc Nat Acad Sci USA 105:15576-15580

Kozmic Z, Ruzickova J, Joasova K, Matsumoto Y, Vopalensky P, Kozmikova I, Strnad H, Kawamura S, Piatigorsky J, Paces V, Vlcek C (2008) Assembly of the cnidarian camera-type eye from vertebrate-like components. Proc Nat Acad Sci USA 105:8989-8993

Krishna VR, Alexander KR, Peachey NS (2002) Temporal properties of the mouse cone electroretinogram. J Neurophysiol 87:42-48

Land MF, Nilsson DE (2006) General-purpose and special-purpose visual systems. In: Warrant EJ, Nilsson DE (eds) Invertebrate vision. Cambridge University Press, Cambridge, pp 167-210

Laughlin SB, Weckström M (1993) Fast and slow photoreceptors-a comparative study of the functional diversity of coding and conductances in Diptera. J Comp Physiol A 172:593-609

Maximov VV (2000) Environmental factors which may have led to the appearance of colour vision. Philos Trans R Soc Lond 355:12391242

Nilsson DE, Coates MM, Gislén L, Skogh C, Garm A (2005) Advanced optics in a jellyfish eye. Nature 435:201-205

Parkefelt L, Nilsson DE, Ekström P (2005) A bilaterally symmetric nervous system in the rhopalia of a radially symmetric cubomedusa. J Comp Neurol 492:251-262

Ruck P (1961a) Electrophysiology of insect dorsal ocellus. 1. Origin of components of electroretinogram. J Gen Physiol 44:605-627

Ruck P (1961b) Electrophysiology of insect dorsal ocellus. 3. Responses to flickering light of dragonfly ocellus. J Gen Physiol 44:641-657 
Skogh C, Garm A, Nilsson DE, Ekström P (2006) The bilateral symmetric rhopalial nervous system of box jellyfish. J Morphol 267:1391-1405

Srinivasan MV, Bernard GD (1975) The effect of motion on visual acuity of the compound eye: a theoretical analysis. Vision Res 15:515-525

Stewart SE (1996) Field behavior of Tripedalia cystophora (class Cubozoa). Mar Freshw Behav Physiol 27:175-188

Viswanathan S, Frishman L, Robson J (2002) Inner-retinal contributions to the photopic sinusoidal flicker electroretinogram of macaques. Doc Opthalmol 105:223-242
Warrant EJ (2004) Vision in the dimmest habitats on Earth. J Comp Physiol A 190:765-789

Warrant EJ, Kelber A, Gislén A, Greiner B, Ribi W, Wcislo WT (2004) Nocturnal vision and landmark orientation in a tropical halictid bee. Curr Biol 14:1309-1318

Weber C (1982a) Electrical activities of a type of electroretinogram recorded from the ocellus of a jellyfish, Polyorchis penicillatus (Hydromedusae). J Exp Zool 223:231-243

Weber C (1982b) Electrical activity in response to light of the ocellus of the hydromedusan, Sarsia tubulosa. Biol Bull 162:413-422 